\title{
Iodine-131 Rituximab Radioimmunotherapy: Durable Control of Follicular Lymphoma
}

Paul C Kruger ${ }^{1}$, David JL Joske ${ }^{2}$ and Harvey Turner $\mathbf{J}^{3 *}$

${ }^{1}$ Department of Hematology, The Queen Elizabeth II Medical Center, Nedlands, Western Australia

${ }^{2}$ The University of Western Australia, Crawley, Western Australia

${ }^{3}$ Department of Nuclear Medicine, Fremantle Hospital and Health Service, Fremantle, Western Australia

*Corresponding author: Harvey Turner J, Director, Department of Nuclear Medicine, Fremantle Hospital and Health Service, Fremantle, Western Australia, Tel: 6108 9431 2888; Fax: 61089431 2889; E-mail: Harvey.Turner@health.wa.gov.au

Received date: Jul 30, 2014, Accepted date: Sep 23, 2014, Publication date: Sep 26, 2014

Copyright: ( 2014 Kruger PC, et al. This is an open-access article distributed under the terms of the Creative Commons Attribution License, which permits unrestricted use, distribution, and reproduction in any medium, provided the original author and source are credited.

\section{Abstract}

Aims: We evaluated the response and toxicity, after long-term follow up of lodine-131 rituximab radioimmunotherapy in patients with follicular lymphoma under the routine clinical care of a single hematologist over a period of 12 years.

Materials and methods: Patients received ${ }^{131}$ I-rituximab radioimmunotherapy according to a standard, personalized dosimetry protocol predicated upon a prescribed whole body radiation absorbed dose of $0.75 \mathrm{~Gy}$. Four doses of maintenance rituximab were subsequently administered over 12 months.

Results: Response rate was $97 \%$ with $24(77 \%)$ patients experiencing a complete remission confirmed on ${ }^{18} \mathrm{~F}-$ fluorodeoxyglucose positron emission tomography-computerized tomography scan. The cohort of 3 patients with duodenal lymphoma all achieved complete remission lasting 4-5 years.

Disclosure statement: There is no conflict of interest to declare with the publication of this work. No author has a financial incentive associated with the publication of this article.

Conclusion: ${ }^{131}$ I-rituximab radioimmunotherapy is an effective, safe, affordable, repeatable treatment which does not compromise future therapy options upon relapse. It is practical, being administered on an outpatient basis, and referring physicians maintain governance of their patients.

Keywords: Iodine-131-rituximab radioimmunotherapy; Duodenal lymphoma; /Indolent NHL

\section{Introduction}

Follicular lymphoma (FL) is the most common form of indolent B cell non-Hodgkin lymphoma (NHL) and accounts for approximately $20 \%$ of lymphomas in adults [1,2]. The Follicular Lymphoma International Prognostic Index (FLIPI) prognostication scheme predicts survival based on the factors age $>60$ years, Ann-Arbor stage $\geq 3$, nodal sites $\geq 5$, hemoglobin $<120 \mathrm{~g} / \mathrm{L}$, and increased lactate dehydrogenase [3]. Asymptomatic patients may be simply observed in the first instance, since systemic therapies are not recognised as curative $[1,4,5]$. As time progresses, remissions post-treatment become shorter and the disease may transform to high grade lymphoma [6,7]. Overall, the median survival of FL ranges from 8 to 12 years [5].

Radioimmunotherapy is an effective and practical treatment option for relapsed/refractory FL given the inherent radiosensitivity of lymphoma. The mechanism of action after systemic administration of a radioimmunoconjugate which binds to the CD20 positive cells, delivers targeted cytotoxic radiation to the lymphoma cell. In addition the 200-cell diameter range of beta rays from Iodine-131 irradiates tumor cells in proximity, even if they do not express the CD20 antigen [8]. We have administered iodine-131 rituximab radioimmunotherapy $\left({ }^{131}\right.$ I-rituximab RIT) to treat patients with relapsed or refractory indolent non-Hodgkin lymphoma, most of whom had follicular lymphoma, with an objective overall response rate of $76 \%$ with a 4 year actuarial survival rate of $59 \% \pm 10 \%$ with minimal toxicity [8]. We reported our clinical experience of the same treatment in 142 consecutive patients with relapsed low grade non-Hodgkin lymphoma, predominantly $\mathrm{FL}$, where objective response rate (ORR) of $67 \%$, complete remission rate (CR) of $50 \%$, and 8 year overall survival (OS) of $48 \%$ were observed [9]. We have also treated a cohort of $68 \mathrm{FL}$ patients first-line with 131I-rituximab radioimmunotherapy achieving 98\% ORR and $89 \%$ CR in a prospective Phase II clinical trial [10].

We describe here the long-term follow-up of patients with FL treated with ${ }^{131}$ I-rituximab RIT, coupled with maintenance rituximab, under the routine clinical care of a single hematologist.

\section{Patients and Methods}

All patients had histologically confirmed FL, an Eastern Cooperative Oncology Group performance status of less than 3, life expectancy of more than 3 months and had received no rituximab within the 6 months prior to ${ }^{131}$ I-rituximab RIT. Rituximab was administered to saturate non-specific binding sites. Radioimmunotherapy with ${ }^{131}$ I-rituximab RIT was then administered on an outpatient basis according to the standard personalised 
Page 2 of 5

dosimetry protocol predicated upon a whole body radiation absorbed dose of $0.75 \mathrm{~Gy}$ [8]. Four doses of maintenance rituximab were administered at 3 month intervals following 131I-rituximab RIT. All patients remained under the governance of the referring Hematologist and the role of the Nuclear Medicine Physician was to perform the dosimetry and to administer the radioimmunotherapy. Administration of 131I-rituximab RIT was performed under Compassionate Patient Use provisions of the Special Access Scheme of the Therapeutic Goods Administration of the Commonwealth Government of Australia on the written informed consent of each patient in accordance with protocols approved by the Human Research Ethics Committee of Fremantle Hospital in conformity with the Declaration of Helsinki and NH\&MRC guidelines. Response to treatment was assessed with $18 \mathrm{~F}$-fluorodeoxyglucose positron emission tomography scan combined with computerized tomography scan $\left({ }^{18} \mathrm{~F}-\mathrm{FDG}-\mathrm{PET}-\mathrm{CT}\right)$ at 3-6 months.

The primary outcome of this study was to assess toxicity, response to treatment, and survival by OS and progression free survival (PFS). Kaplan-Meier methodology was used to estimate median OS and PFS.

\section{Results}

Thirty one patients with FL presenting in the course of routine clinical practice of a single Hematologist were referred for treatment with 131I-rituximab RIT between 2001 and 2013. Baseline patient and disease characteristics are shown in Table 1 . There were 14 male and 17 female patients with a median age of 54 years (range 30-74). Sites of disease included nodal disease only; 18/31(58\%), nodal plus extranodal disease; 10/31(32\%), and duodenal disease only; 3/31(10\%). AnnArbor stage III-IV disease was evident in 18 patients (58\%). The FLIPI Score was 0 in 7/31(23\%), 1 in 13/31(42\%), 2 in 4/31(13\%), 3 in $6 / 31(19 \%)$, and 4 in $1 / 31(3 \%)$. The median time from diagnosis to 131I-rituximab RIT was 39 months (range 2-354). Seven patients had 131I-rituximab RIT as first-line treatment. Twenty-four patients had had treatment prior to 131I-rituximab RIT, and the median number of prior lines of therapy was two. Prior therapy modalities included radiation only in $1(3 \%)$ patient, with the others having a variety of combination chemotherapy regimens, including rituximab, cyclophosphamide, doxorubicin, vincristine, prednisolone (R-CHOP), rituximab, fludarabine, mitoxantrone, dexamethasone (R-FMD), cyclophosphamide, etoposide, vincristine, prednisolone (CEOP), prednisolone, chlorambucil, cyclophosphamide. One patient had had previous myeloablative chemotherapy and autologous hematopoietic stem cell transplantation (ASCT). Treatment was indicated in every patient as per the Groupe pour l'Etude de Lymphome Folliculaire (GELF) criteria [11], being symptomatic in 30/31 (97\%), 8/31(26\%) had high tumour bulk, and $4 / 31(13 \%)$ had a lactate dehydrogenase above the upper limit of normal.

\begin{tabular}{|l|l|}
\hline Parameter & $\mathbf{n}(\%)$ \\
\hline Gender & male $14(45 \%)$, female $17(55 \%)$ \\
\hline Main site & $\begin{array}{l}18(58 \%) \\
10(32 \%) \\
3(10 \%)\end{array}$ \\
\hline $\begin{array}{l}\text { Lymph node disease only } \\
\text { Dumph node plus other site }\end{array}$ & $7(23 \%)$ \\
\hline FLIPI & \\
\hline 0 & \\
\hline
\end{tabular}

\begin{tabular}{|l|l|}
\hline 1 & $13(42 \%)$ \\
2 & $4(13 \%)$ \\
4 & $6(19 \%)$ \\
$1(3 \%)$ \\
\hline $\begin{array}{l}\text { Time from diagnosis to 1311-rituximab RIT } \\
\text { (months) }\end{array}$ & median 39, range2-354 \\
\hline Number of patients given prior treatment & $24(77 \%)$ \\
\hline Number of prior lines of therapy & \\
\hline 1 & 13 \\
2 & 10 \\
3 & 1 \\
\hline
\end{tabular}

Table 1: Patient and disease characteristics at diagnosis. FLIPI follicular lymphoma international prognostic index; ${ }^{131}$ I-rituximab RIT - iodine-131 rituximab radioimmunotherapy.

At the time of writing, 30/31(97\%) patients are alive. Table 2 summarises treatment outcomes. The median OS has not been reached after a median follow up of 65 months. The median PFS is 71 months (range 6-152) (Figure 1). The ORR was 30/31(97\%), CR confirmed on 18F-FDG-PET-CT in 24/31(77\%), partial response (PR) in $4 / 31(13 \%)$. Unconfirmed complete remission (CRu) was seen in $2 / 31(7 \%)$, and progressive disease (PD) in $1 / 31(3 \%)$. There were no acute hypersensitivity reactions during the infusion of 131I-rituximab RIT and no infections or episodes of bleeding requiring hospital admission. Grade III-IV neutropenia (defined as absolute neutrophil count $<1 \times 109 / \mathrm{L})$ occurred in $4 / 31(13 \%)$ patients. Grade III-IV thrombocytopenia (defined as platelets <50x109/L) occurred in $1 / 31(3 \%)$ patient. Subclinical hypothyroidism occurred in $4 / 31(13 \%)$ patients and was treated. Myelodysplasia occurred in only one patient.

\begin{tabular}{|l|l|}
\hline Parameter & $\mathbf{n}(\%)$ \\
\hline Response to ${ }^{131}$-rituximab RIT & \\
\hline CR on ${ }^{18}$ F-FDG-PET-CT & $24(77 \%)$ \\
CRu & $2(7 \%)$ \\
PR on ${ }^{18}$ F-FDG-PET-CT & $4(13 \%)$ \\
PD & $1(3 \%)$ \\
\hline Toxicity & \\
\hline Atypical infection & $0(0 \%)$ \\
Neutropenia $<1 \times 109 / L$ & $4(13 \%)$ \\
Thrombocytopenia $<50 \times 109 / L$ & $1(3 \%)$ \\
Hypothyroidism & $4(13 \%)$ \\
Myelodysplasia & $1(3 \%)$ \\
\hline PFS (months) & median 71, range 6-152 \\
\hline OS (months) & Not reached after a median follow \\
& up of 65 months, range 9-152 \\
\hline
\end{tabular}

Table 2: Response to treatment, toxicity, survival. CR - complete remission; $\mathrm{CRu}$ - unconfirmed complete remission; $\mathrm{PR}$ - partial response; 18F-FDG-PET-CT - Fluorine-18-fluorodeoxyglucose positron emission tomography combined with computerized tomography; PD - progressive disease; PFS - progression-free survival; OS - overall survival. 


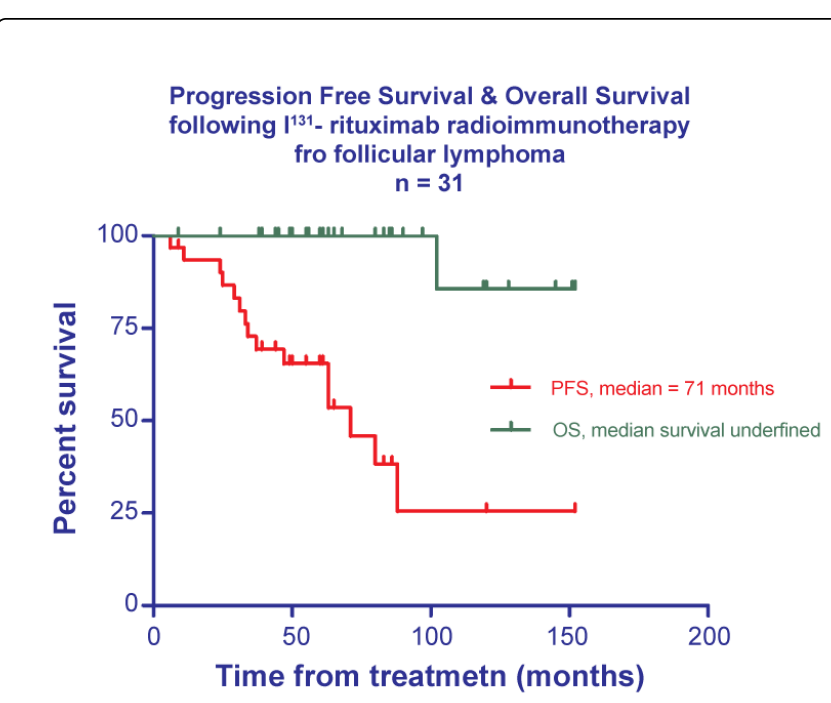

Figure 1: Kaplan-Meier plot of progression free survival (PFS) and overall survival (OS) for 31 patients following 131I-rituximab radioimmunotherapy for follicular lymphoma.

\section{Discussion}

The choice of treatment modality of NHL depends in part upon the stage of disease and the patients' ability to tolerate it. Radiation therapy is effective for stage I-II disease and can lead to long lasting remissions [12]. Anti-CD20 monoclonal antibody therapy such as rituximab either alone or with combination chemotherapy is effective for stage III-IV disease [13]. Chemoimmunotherapy with R-CHOP is still widely used as efficacy is established with an overall response rate of approximately $90 \%$. Newer agents such as bendamustine are emerging but have yet to be proven [2]. Some prefer to withhold anthracyclines, so that it is available later in the event of disease transformation. Autologous or allogeneic hematopoietic stem cell transplantation is reserved for selected patients in high risk situations such as relapsed/refractory or transformed disease [2,4]. The survival of patients with FL is improving as a result of the sequential application of effective therapies, particularly rituximab, including 131I-rituximab RIT, and improved supportive care [4].

Reporting a cohort of NHL patients from a single hematologist experience has obvious limitations but is representative of real-world clinical practice over a decade. Our patients represent a heterogeneous group and there was variation in the timing of 131I-rituximab RIT (first-line vs salvage therapy), and the stage of disease. All patients required treatment according to GELF criteria (single lesion $>7 \mathrm{~cm}$, three nodal sites $>3 \mathrm{~cm}$, splenomegaly, cytopenias, effusions, organ compression). Over one third (13/31) of our patients have achieved more than 5 years relapse-free survival. The choice of 131I-rituximab RIT as the main therapy was taken after meticulous in-house development, calculation of individual dosimetry, and extensive published evidence of efficacy $[8,9,10,14]$.

Radioimmunotherapy is a relatively recent therapeutic modality for $\mathrm{FL}$ and has the potential to change the approach to the management of this heterogeneous disease. Commercially available murine antibody based agents yttrium-90 ibritumomab-tiuxetan and iodine-131 tositumomab have been approved for the treatment of relapsed and refractory FL and show potent single-agent activity; 131I-tositumomab treatment had an ORR of 95\%, CR 75\%, actuarial 5-year PFS 59\%, and median PFS 6.1 years in a phase II study of 76 consecutive patients with previously untreated grade I-II, Ann-Arbor stage III-IV FL [15]. However, sale of this agent, was discontinued and marketing approval withdrawn in the United States in February 2014, consequent on poor sales attributed to physician reimbursement issues rather than any proven lack of efficacy [16]. Yttrium-90 ibritumomab-tiuxetan treatment had an ORR of $87 \%$, CR or CRu $56 \%$, and after a median follow-up of 30.6 months the median PFS for all patients was 25.9 months and median OS had not been reached in patients with previously untreated FL [17]. Furthermore, 90Y-ibritumomabtiuxetan treatment produces statistically significant higher ORR and $\mathrm{CR}$ compared to rituximab alone in patients with relapsed or refractory low-grade FL [18]. However, toxicity in clinical practice has been reported to be relatively high with grade 3-4 neutropenia in 55\% and thrombocytopenia in 56\% [19]. In clinical practice rates of response to treatment with $90 \mathrm{Y}$-ibritumomab tiuxetan and ${ }^{131} \mathrm{I}$ tositumomab are at the lower range of those reported in the literature for various reasons, with an overall response rate of $47 \%$ and complete response rate $13 \%$ [19]. On the contrary, our group of patients in routine clinical practice achieved an ORR of $97 \%$ and CR (confirmed on ${ }^{18} \mathrm{~F}$-FDG-PET-CT) of $77 \%$ post ${ }^{131}$ I-rituximab RIT.

Duodenal FL without nodal disease is rare but occurred in 3 of our patients who all had symptoms attributable to bowel wall lymphoma. All achieved CR confirmed on ${ }^{18} \mathrm{~F}$-FDG-PET-CT. These patients continue to be asymptomatic at 44,55, and 60 months follow-up post 131I-rituximab RIT and four doses of maintenance rituximab, and none have required further treatment. This cohort has achieved excellent response, but this should be interpreted with caution as it seems to be a remarkably indolent FL variant more likely than nodal FL to present with early stage disease [20]. Duodenal FL has different features from nodal FL, including grade 1/2 histology, no marginal zone or plasmacytic differentiation, no reactive germinal centres, and the $t(14 ; 18)(\mathrm{q} 32 ; \mathrm{q} 21)$ positive B-cells do not acquire further karyotypic changes thus they have a low malignant potential [20]. The gene expression profile shows upregulation of Chemokine ligand 20 (CCL20) and mucosal vascular addressin cell adhesion molecule 1 (MAdCAM-1) which is downregulated in nodal FL and may play a role in molecular pathogenesis which is presently unclear [21].

As FL is highly treatable but ultimately incurable, it is essential to treat patients with effective therapies which are tolerable. Rituximab is an attractive monoclonal antibody as it preferentially treats B cell lymphoma without affecting $\mathrm{T}$ cell function. Other advantages of ${ }^{131} \mathrm{I}$ rituximab RIT are the ability to repeat administration in the event of relapse, given the lack of potential for human anti-mouse antibody formation, which may occur in 131I-tositumomab radioimmunotherapy [22]. Outpatient administration of 131Irituximab RIT is safe and the median radiation exposure is within the limits recommended by international guidelines [23]. Patient and carer issues with outpatient radiation safety of 131I-rituximab RIT have also been recently addressed in depth [24]. Our treatment constituted full combination radioimmunotherapy, where the patient received treatment with a full course of rituximab immunotherapy at standard dosage, followed by radioimmunotherapy and maintenance rituximab. Potential toxicity of ${ }^{131}$ I-rituximab RIT includes selflimited neutropenia and thrombocytopenia, and subclinical hypothyroidism. In our reported experience of 142 consecutive patients, grade IV neutropenia occurred in $10 \%$, grade IV thrombocytopenia in $6 \%$, and grade IV anemia in $1 \%$ [9]. In the 
current single physician study, toxicity included grade III-IV neutropenia in $13 \%$, grade III-IV thrombocytopenia in $3 \%$, which compares favourably with conventional R-CHOP chemotherapy regimen [25]. One of our patients was diagnosed with myelodysplasia on a bone marrow aspirate and trephine after becoming pancytopenic 8 years post 131I-rituximab RIT. Cytogenetic analysis of bone marrow aspirate showed an abnormal complex and mosaic karyotype consistent with myelodysplasia, most likely due to prior chemotherapy with chlorambucil-prednisolone, melphalan- cyclophospamideprednisolone, 4 cycles R-CHOP. Compromise of hematopoietic stem cell collection in patients treated with prior radioimmunotherapy may be a potential concern, which can be addressed by a second and/or salvage harvest. In our small experience, stem cell collection has not been impaired after ${ }^{131}$ I-rituximab RIT and patients have undergone autologous stem cell transplantation with no significant difference in engraftment kinetics when compared to patients treated with prior chemotherapy $[10,26]$.

\section{Conclusion}

In real life clinical practice, epitomized by a single hematologist's clinical experience, durable control of FL by ${ }^{131}$ I-rituximab RIT is achievable without significant toxicity in non-selected patients, including those pretreated with chemotherapy. Subsequent therapeutic options are not compromised upon relapse and outpatient ${ }^{131} \mathrm{I}$ rituximab RIT is practical, affordable, and preserves quality of life.

\section{Acknowledgements}

The authors wish to thank Anna Chiam for clinical data management, Suet Mei Yu for statistical analysis and Jenny Lavin for preparation of the manuscript.

No grant funding or Pharma support was solicited or received.

\section{References}

1. Gribben JG (2007) How I treat indolent lymphoma. Blood 109: 4617-4626.

2. Ujjani C, Cheson BD (2013) The optimal management of follicular lymphoma: an evolving field. Drugs 73: 1395-1403.

3. Solal-Céligny P, Roy P, Colombat P, White J, Armitage JO, et al. (2004) Follicular lymphoma international prognostic index. Blood 104: 1258-1265.

4. Swenson WT, Wooldridge JE, Lynch CF, Forman-Hoffman VL, Chrischilles E, et al. (2005) Improved survival of follicular lymphoma patients in the United States. J Clin Oncol 23: 5019-5026.

5. Reiser M, Diehl V (2002) Current treatment of follicular non-Hodgkin's lymphoma. Eur J Cancer 38: 1167-1172.

6. Buchegger F, Larson SM2, Mach JP3, Chalandon Y4, Dietrich PY5, et al. (2013) Radioimmunotherapy combined with maintenance anti-CD20 antibody may trigger long-term protective $\mathrm{T}$ cell immunity in follicular lymphoma patients. Clin Dev Immunol 2013: 875343.

7. Bodet-Milin C, Ferrer L, Pallardy A, Eugène T, Rauscher A, et al. (2013) Radioimmunotherapy of B-Cell Non-Hodgkin's Lymphoma. Front Oncol 3: 177.

8. Leahy MF, Seymour JF, Hicks RJ, Turner JH (2006) Multicenter phase II clinical study of iodine-131-rituximab radioimmunotherapy in relapsed or refractory indolent non-Hodgkin's lymphoma. J Clin Oncol 24: 4418-4425.

9. Leahy MF, Turner JH (2011) Radioimmunotherapy of relapsed indolent non-Hodgkin lymphoma with 131I-rituximab in routine clinical practice: 10-year single-institution experience of 142 consecutive patients. Blood 117: 45-52.
10. McQuillan AD, Macdonald WB, Turner JH (2014) Phase II study of firstline 131I-rituximab radioimmunotherapy in follicular non-Hodgkin lymphoma and prognostic 18F-fluorodeoxyglucose positron emission tomography. Leuk Lymphoma .

11. Brice P, Bastion Y, Lepage E, Brousse N, Haïoun C, et al. (1997) Comparison in low-tumor-burden follicular lymphomas between an initial no-treatment policy, prednimustine, or interferon alfa: a randomized study from the Groupe d'Etude des Lymphomes Folliculaires. Groupe d'Etude des Lymphomes de l'Adulte. J Clin Oncol 15: $1110-1117$

12. Haas RL, Poortmans P, de Jong D, Aleman BM, Dewit LG, et al. (2003) High response rates and lasting remissions after low-dose involved field radiotherapy in indolent lymphomas. J Clin Oncol 21: 2474-2480.

13. Feuring-Buske M, Kneba M, Unterhalt M, Engert A, Gramatzki $M$, et al. (2000) IDEC-C2B8 (rituximab) anti-CD20 antibody treatment in relapsed advanced-stage follicular lymphomas: results of a phase-II study of the German Low-Grade Lymphoma study group. Annuals of Hematology 79: 493-500.

14. Boucek JA, Turner JH (2005) Validation of prospective whole-body bone marrow dosimetry by SPECT/CT multimodality imaging in 131I-antiCD20 rituximab radioimmunotherapy of non-Hodgkin's lymphoma. European Journal of Nuclear Medicine and Molecular Imaging 32: 458-469.

15. Kaminski MS, Tuck M, Estes J, Kolstad A, Ross CW, et al. (2005) 131Itositumomab therapy as initial treatment for follicular lymphoma. N Engl J Med 352: 441-449.

16. http://www.xconomy.com/national/2013/08/26/why-good-drugssometimes-fail-in-the-market-the-bexxar-story/

17. Scholz CW, Pinto A, Linkesch W, Linden O, Viardot A, et al. (2013) 90Yttrium-ibritumomab-tiuxetan as first-line treatment for follicular lymphoma: 30 months of follow-up data from an international multicenter phase II clinical trial. Journal of Clinical Oncology 31: 308-313.

18. Witzig TE, Gordon LI, Cabanillas F, Czuczman MS, Emmanouilides C, et al. (2002) Randomized controlled trial of yttrium-90 labeled ibritumomab tiuxetan radioimmunotherapy versus rituximab immunotherapy for patients with relapsed or refractory low-grade, follicular, or transformed B-cell non-Hodgkin's lymphoma. Journal of Clinical Oncology 20: 2453-2463.

19. Jacene HA, Filice R, Kasecamp W, Wahl RL (2007) Comparison of 90Yibritumomab tiuxetan and 131I-tositumomab in clinical practice. J Nucl Med 48: 1767-1776.

20. Schmatz AI, Streubel B, Kretschmer-Chott E, Puspok A, Jager U, et al. (2011) Primary follicular lymphoma of the duodenum is a distinct mucosal/submucosal variant of follicular lymphoma: a retrospective study of 63 cases. Journal of Clinical Oncology 29: 1445-1451.

21. Takata K, Tanino M, Ennishi D, Tari A, Sato Y, et al. (2014) Duodenal follicular lymphoma: comprehensive gene expression analysis with insights into pathogenesis. Cancer Sci 105: 608-615.

22. Buchegger F, Antonescu C, Delaloye AB, Helg C, Kovacsovics T, et al. (2006) Long-term complete responses after 131I-tositumomab therapy for relapsed or refractory indolent non-Hodgkin's lymphoma. Br J Cancer 94: 1770-1776.

23. Calais PJ, Turner JH (2012) Outpatient 131I-rituximab radioimmunotherapy for non-Hodgkin lymphoma: a study in safety. Clin Nucl Med 37: 732-737.

24. Calais PJ, Page AC, Turner JH (2012) Management of fear of radiation exposure in carers of outpatients treated with iodine-131. Ann Nucl Med 26: 508-514.

25. Hiddemann W, Kneba M, Dreyling M, Schmitz N, Lengfelder E, et al. (2005) Frontline therapy with rituximab added to the combination of cyclophosphamide, doxorubicin, vincristine, and prednisone (CHOP) significantly improves the outcome for patients with advanced-stage follicular lymphoma compared with therapy with $\mathrm{CHOP}$ alone: results of a prospective randomized study of the German Low-Grade Lymphoma Study Group. Blood 106: 3725-3732. 
Citation: Kruger PC, Joske DJL, Turner JH (2014) lodine-131 Rituximab Radioimmunotherapy: Durable Control of Follicular Lymphoma. J Nucl Med Radiat Ther 5: 191. doi:10.4172/2155-9619.1000191

Page 5 of 5

26. Derenzini E, Stefoni V, Maglie R, Casadei B, Pellegrini C, et al. (2013) Collection of hematopoietic stem cells after previous radioimmunotherapy is feasible and does not impair engraftment after autologous stem cell transplantation in follicular lymphoma. Biology of Blood Marrow Transplantation 19: 1695-1701. 